\title{
Consumo de drogas y alcohol: de la agenda pública a la agenda social de los gobiernos subnacionales. La experiencia de la provincia de Santa Fe, Argentina
}

\section{Consumption of drugs and alcobol: from the subnational government public agenda to the social one. The experience of Santa Fe province, Argentina}

\author{
Nieto, Cecilia; Sartor Schiavoni, María Laura
}

Cecilia Nieto Sobre la autora ceci4nieto@gmail.com Universidad Nacional de Rosario, Argentina María Laura Sartor Schiavoni Sobre la autora malaura.sartor@gmail.com Universidad Nacional de Rosario, Argentina

PAPELES del Centro de Investigaciones de la Facultad de Ciencias Jurídicas y Sociales de la UNL Universidad Nacional del Litoral, Argentina

ISSN: $1853-2845$

ISSN-e: 2591-2852

Periodicidad: Semestral

vol. 12, núm. 23, 2021

papelesdelcentro@fcjs.unl.edu.ar

Recepción: 30 Agosto 2021

Aprobación: 15 Septiembre 2021

URL: https://doi.org/10.14409/p.v12i23.10784

\section{(1) (2)}

Esta obra está bajo una Licencia Creative Commons AtribuciónNoComercial-CompartirIgual 4.0 Internacional.
Resumen: Las problemáticas vinculadas al consumo de drogas forman parte de la agenda social y política actual. No obstante, este tema se presenta en la agenda de forma polisémica y en consecuencia, se entrecruzan los ámbitos punitivos, de la salud y sociales. El consumo de sustancias es un fenómeno de carácter global que, no obstante, adquiere características propias en cada territorio. Las ciudades se ubican como un primer eslabón de contacto con la población, por lo cual los gobiernos de los ejidos urbanos se ven en la necesidad de responder ante la problemática, interpelados por la cercanía de como la viven. En este trabajo nos proponemos, en primer lugar, brindar un diagnóstico sobre los debates actuales de los abordajes de las problemáticas del consumo de drogas y alcohol. En segundo lugar buscaremos, recuperar aprendizajes, a partir de la experiencia de la Agencia de Prevención de Consumo de Drogas y Tratamiento Integral de las Adicciones, APRECOD, del Ministerio de Gobierno y Reforma del Estado de la Provincia de Santa Fe durante el período 2017-2019, dando cuenta del rol central que tienen los gobiernos subnacionales en la construcción de políticas sociales urbanas, que den respuesta al abordaje integral de la problemática.

Palabras clave: políticas sociales, gobiernos locales, integralidad, prevención, consumo de sustancias.

Abstract: The problematics associated to the consumption of drugs are part of the social agenda and actual policies of the states. However, this subject is presented in the agenda in a polysemic way, therefore the punitive health and social areas are tightly entwined. The consumption of substances is a global phenomenon that acquires its oun features in each territory. The cities stand as the first link of contact with the urban population, this is why the governments of urban areas are exposed to the necessity of providing real answers to this increasing problematic that is also lived very closely by the whole community. In carrying out this work we intend to 1 st provide an accurate diagnosis of current debates on the approaches to the problematics of drugs and alcohol consumption. In 2nd place we'll focus on recovering learning experiences from The Agency for the Prevention of Drug Use and integrated treatment to Addictions (APRECOD), 
belonging to the Ministery of Governments and Reform of the State of Santa Fe Province, during the years 2017 of 2019. Highlighting the mail role that subnational governments have in the building of social urban policies that should give answers to an integrated approach of this complex problematic.

Keywords: social policies, local government, prevention, consumption of substances, integrated approach.

\title{
1. Introducción: las políticas de drogas en la agenda de las políticas sociales urbanas
}

\author{
"Creo que las drogas han destruido muchas vidas, pero las malaspoliticas de los gobiernos \\ han destruido muchas más"
}

Kofi Annan - Ex Secretario general de la ONU y premio Nobel de la Paz.

Los problemas vinculados al consumo de drogas se han modificado considerablemente en las últimas décadas. Los reportes de organismos internacionales, como la Comisión Interamericana para el Control del Abuso de Drogas (CICAD-OEA) y la Oficina de Naciones Unidas contra las Drogas (ONUDD) dan cuenta de situaciones alarmantes vinculadas a los consumos, dado que no sólo hay un aumento de la población consumidora y una mayor cantidad de sustancias disponibles en el mercado, sino que lo que resulta más preocupante para los estudios de la temática y los gobiernos que deben emprender políticas públicas es que hay una disminución en la perspectiva de riesgo en la población frente al uso de drogas (Gonzalez et al., 2017, p. 4).

Argentina y la provincia de Santa Fe no escapan a esta realidad, mostrando distintos efectos e impactos sociales y sanitarios en los diversos territorios. Sin embargo, si bien es una problemática incorporada en la agenda pública, su inclusión en la agenda de las políticas sociales urbanas ha estado ralentizada por diversos factores que, al mismo tiempo, inciden en la forma en que la problemática se presenta.

En primer lugar, podemos mencionar dos aspectos centrales que permiten, al menos en parte, explicar cómo se ha definido el problema y las respuestas estatales, y no estatales, que se han brindado. En primer lugar, una de las causas centrales es la convivencia de distintos paradigmas de abordaje, que muchas veces han traccionado para que la problemática sea tomada por ámbitos de la seguridad o de especialistas, por fuera de la órbita del Estado. En segundo lugar, y como reflejo del primer punto, nos encontramos con la convivencia de marcos legales que tensionan entre sí, léase la Ley Nacional de Tenencia y Tráfico de Estupefacientes (23.737) del año 1989, que penaliza todo consumo de sustancias ilegales, y la Ley Nacional de Salud Mental (26.657), del año 2010, que incorpora en su artículo 4 a las adicciones dentro del campo de la salud mental y por ende, dentro de las políticas sanitarias, más allá de la legalidad de la sustancia que se consuma y abordando a la persona que consume como un sujeto de derecho.

Considerando estos dos aspectos, la primera parte de este trabajo recorre los principales conceptos y paradigmas, así como algunos puntos centrales del marco regulatorio, a fines de presentar la problemática. 
El segundo momento de este trabajo, se centra en las políticas públicas de drogas y su inserción en las políticas sociales urbanas. Es menester destacar que desde 1980 Argentina viene atravesando un proceso de descentralización de sus políticas sociales (Repetto, 2010, p.13), pasando a tomar mayor protagonismo el lugar de las provincias y los municipios. Sin embargo este proceso no ha sido homogéneo en todos los campos, ni se ha desarrollado de forma lineal.

En cuanto a las políticas de drogas en el ámbito nacional, si bien la Secretaría de Políticas Integrales de Drogas (SEDRONAR) fue creada en 1989, es decir que posee más de treinta años de creación como Secretaría de Estado, ha estado atravesada por cambios profundos. Estos se reflejan, por ejemplo, en su viraje de "Secretaría de Programación para la Prevención de la Drogadicción y la Lucha contra el Narcotráfico", con mirada puesta en la seguridad y reducción de la oferta, a su actual enfoque centrado en la reducción de la demanda y la salud pública. Sumado a esto, también hubo discontinuidades en las autoridades, que conllevaron marcadas diferencias en su forma de intervenir sobre el problema. Estos cambios se tradujeron en una fragilidad de las políticas públicas para abordar la temática que no se han podido traducir en políticas de Estado basadas en evidencia y a largo plazo, así como en iniciativas que descentralicen la política nacional en las provincias y los municipios.

En cuanto a los gobiernos subnacionales, entendemos que en los últimos treinta años han ido asumiendo mayor protagonismo en cuanto a las agendas sociales. Se han convertido en actores fundamentales, capaces por su cercanía a los ciudadanos y el territorio, de ser los más adecuados para planificar y ejecutar políticas sociales integrales tal como hoy lo demanda la complejidad de la problemática. Sin embargo, existe una carencia de literatura que recoja estas experiencias en los niveles provinciales y municipales. Uno de los antecedentes recientes que trabajan la problemática en específico es la publicación de Sedronar "Municipios en Acción. Fundamentos y estrategias para una política local sobre drogas", publicado en 2018, y que sirve como antecedente a este trabajo.

Es en este sentido, que en este artículo nos proponemos reflexionar, a través de la experiencia santafesina, sobre el rol de las ciudades como ámbitos estratégicos para el diseño e implementación de políticas de abordaje de los consumos problemáticos; y de sus gobiernos, como actores capaces de abordar la complejidad de nuevos problemas sociales.

En la actualidad, con el creciente protagonismo de las ciudades, lo urbano y lo social se mezclan. "Lo urbano, en su renovada dimensión territorial, es el espacio en el que se mueven las políticas, buscando respuestas específicas para ese espacio" (Subirats y Montaner, 2012, p.17). En este sentido, las políticas urbanas ya no refieren únicamente a las políticas urbanísticas, o a la ordenación de usos, hábitats y movilidades; sino que nos apoyaremos en una concepción de la política urbana como síntesis transversal del conjunto de políticas en el territorio.

Este trabajo entiende entonces las políticas urbanas "como marco en el que situar actuaciones integrales, pensadas e implementadas desde la proximidad, pero integrando la multiplicidad de mecanismos de intervención multinivel" (Subirats y Montaner, 2012, p.19)

Entendemos que este artículo no se trata de un trabajo acabado, pero que posibilita abrir un campo de preguntas y experiencias que permiten reflexionar 
acerca de los abordajes de los consumos problemáticos de sustancias en las agendas de las políticas sociales urbanas.

\section{Un acercamiento a la problemática de los consumos y sus abordajes: conceptos, paradigmas y marco legal}

Como punto de partida podemos preguntarnos qué decimos cuando hablamos de drogas. La Organización Mundial de la Salud (OMS) define la palabra droga como "toda sustancia terapéutica o no que, introducida en el organismo por cualquier vía de administración, produce una alteración, del natural funcionamiento del sistema nervioso central del individuo y es, además, susceptible de crear dependencia, ya sea psicológica, física o ambas, de acuerdo con el tipo de sustancia, la frecuencia del consumo y la permanencia en el tiempo". Otro término utilizado frecuentemente es el de sustancias psicoactivas, entendido como todo compuesto químico que es capaz de cambiar el modo en que funciona la mente.

A lo largo del documento utilizaremos ambos términos de forma indistinta, sin poner el foco en la legalidad o no de las sustancias o drogas.

Otro concepto clave que abona a la temática es el de consumo problemático de sustancias. Este concepto parte de la idea de que no existe una única forma de entender el consumo, sino que la misma varía según el momento histórico, político, social y económico en el que se presenta. Se enfoca en la relación entre sustancia, el sujeto y el contexto, y desde esta perspectiva se asume el desafío de trabajar la vinculación de estos tres ejes (Aprecod, 2017).

Desde este punto de partida, el modelo de intervención que aquí se adopta es el multidimensional, basado en el paradigma de la complejidad. Desde este posicionamiento es imposible mirar el tema desde un solo enfoque, sino que es necesario hacerlo desde la multicausalidad contextualizada en un tiempo histórico social y en un territorio determinado, habitado de múltiples y diversos actores.

Esta mirada sostiene que necesariamente los tres factores: sustancia, sujeto y contexto se articulan en la configuración del problema. Por lo tanto, no se trata solamente de la sustancia consumida, sino de la persona, sus derechos y el vínculo que establece con la sustancia en un lugar y tiempo determinado (Aprecod, 2017). ${ }^{1}$

Así, este concepto invita a cuestionarse y romper estereotipos simplistas para abarcar el problema en su complejidad, problematizando los modelos que a lo largo del tiempo, y aún en la actualidad, conviven al momento de comprender y abordar la problemática.

\subsection{Modelos y enfoques de abordaje}

Para comprender la disparidad con la que se interviene en la problemática, los disímiles discursos, la diversidad de actores que irrumpen la escena y las incipientes políticas sociales locales, es necesario detenernos en los distintos modelos y enfoques. Los mismos, si bien son planteados por la literatura como 
modelos que han ido presentándose a lo largo del tiempo, conviven en la actualidad, y cada uno de ellos aporta formas de actuar e intervenir.

El primer enfoque, corresponde al modelo ético jurídico. Éste se sustenta en el derecho y la legislación. Se trata de un enfoque punitivo, que entiende al consumo de drogas como un delito y por ende a la persona que consume como un delincuente. Se centra principalmente en la sustancia, "la droga", y sobre todo en aquellas de carácter ilegal. Al establecer un vínculo causal entre consumo y criminalidad no sólo construye un estereotipo en relación a los usuarios, sino que produce y reproduce estigmatización y discriminación. Asimismo, al focalizarse en la sustancia, define también al usuario como víctima de la droga, siendo entonces delincuente y víctima a la vez y construye una demonización o fetichizacio\#n de la sustancia (Defensoría de Niños, Niñas y Adolescentes, 2016).

Es un enfoque centrado en el prohibicionismo y desde el cual se evitará por todos los medios el consumo de sustancias. Por lo tanto, al momento de definir las intervenciones las estrategias más frecuentes son aquellas que buscan la imposibilidad de acceso y disponibilidad a la sustancia; y, por lo tanto, las medidas preventivas se asimilan al control. Desde este enfoque, la problemática debe ser abordada centralmente desde ámbitos de la seguridad, dejando por fuera a las áreas sociales.

Podemos reconocer que este modelo es uno de los más usuales, sobre todo en los discursos de los medios de comunicación, en los cuales se estigmatiza a las personas que consumen, relacionándolas causalmente con situaciones de delincuencia.

El segundo enfoque se centra en la mirada médico-hegemónica. Desde este modelo la persona que consume es considerada un enfermo, al que especialistas, con conocimientos específicos, deben curar para su posterior reinserción social. "Al asimilar la droga como enfermedad, el causante de los problemas se ve como un agente externo, una epidemia, que afecta al cuerpo social" (Defensoría de Niños, Niñas y Adolescentes, 2016).

Desde esta mirada las intervenciones preventivas se enfocan en la perspectiva médico-epistemológica, asimilando el consumo problemático de sustancias a enfermedades infectocontagiosas. En relación al abordaje, la estrategia se centra también en el miedo y el abstencionismo pero, a diferencia del enfoque anterior, hace principal eje en las consecuencias negativas que la ingesta de sustancias tiene sobre el cuerpo.

Los dos modelos expuestos hasta aquí, aunque con perspectivas diferentes, ponen claramente el eje en las drogas o sustancias y se caracterizan por ser modelos causales.

Como superador a estas miradas, en el marco del paradigma de la complejidad, surgen un conjunto de modelos que buscan romper con las mono-causalidades. Desde estos enfoques se presentan un conjunto de intervenciones que buscan superar un paradigma epistemológico individual, reconociendo la complejidad y la necesidad de un trabajo inter y multidisciplinario (Defensoría de Niños, Niñas y Adolescentes, 2016).

Se entiende que los consumos de sustancias psicoactivas hoy deben ser entendido como una problemática compleja que encuentra su origen en una multiplicidad de causas. Por lo tanto, el modelo desde donde abordarla debe ser el de la complejidad y multicausalidad, haciendo eje en las personas como sujetos 
de derechos, en sus contextos y bajo un enfoque de salud pública. Siguiendo estos lineamientos la intervención debe ser pensada como promoción de derechos. Esto implica reconocer y fortalecer las redes sociales y por lo tanto, generar sinergias en la comunidad, entendiendo al sujeto como como sujeto de derechos, partícipe no sólo de la construcción del problema, sino también de las posibles soluciones para sí mismo (Defensoría de Niños, Niñas y Adolescentes, 2016).

Por último, cabe destacar que esta óptica se aleja del modelo prohibicionista y promueve la reducción de riesgo y daños, entendidas como prácticas que "tienen como primer objetivo disminuir los efectos negativos producto del uso de drogas, y a partir de allí, construir conjuntamente con el sujeto objetivos de mayor complejidad, con el fin de mejorar (su) calidad de vida" (sitio web de Asociación Civil Intercambios).

Las estrategias de reducción de daños buscan superar prácticas individuales y comprender las condiciones de vulnerabilidad de los sujetos. Sólo de esta forma podremos realizar intervenciones apropiadas que promuevan relaciones específicas de cuidado y reciprocidad entre los individuos y los grupos.

\subsection{La Ley de Salud Mental como horizonte rector de las politicas de drogas}

En Argentina en relación al consumo de sustancias nos encontramos, con marcos legales que tensionan en cuanto a sus posturas: algunos que criminalizan el consumo de sustancias ilegales y otros que lo toman como parte de las problemáticas de salud, desde un enfoque de derechos.

En el año 2010, con la sanción de la Ley Nacional de Salud Mental (26.657) se realizó un cambio paradigmático en el abordaje de la problemática, dado que la misma, considera, en su artículo 4 que: "Las adicciones deben ser abordadas como parte integrante de las políticas de salud mental. Las personas con uso problemático de drogas, legales e ilegales, tienen todos los derechos y garantías que se establecen en la presente ley en su relación con los servicios de salud".

Posteriormente, la Ley Nacional 26.934, conocida como "Plan integral para el abordaje de los consumos problemáticos", abona en el mismo sentido al definir a los consumos problemáticos como "aquellos consumos que -mediando o sin mediar sustancia alguna- afectan negativamente, en forma crónica, la salud física o psíquica del sujeto, y/o las relaciones sociales".

Estas leyes se alejan de una mirada punitiva y discriminadora con el objetivo de ampliar y complejizar el enfoque y sobre todo poder -en aquellas situaciones que sean necesarias y ante la voluntad de las personas-, garantizar su atención desde abordajes interdisciplinarios. La Ley 26.934 asimismo amplifica la perspectiva al afirmar que "los consumos problemáticos pueden manifestarse como adicciones o abusos al alcohol, tabaco, drogas psicotrópicas -legales o ilegales- o producidos por ciertas conductas compulsivas de los sujetos hacia el juego, las nuevas tecnologías, la alimentación, las compras o cualquier otro consumo que sea diagnosticado compulsivo por un profesional de la salud" (art. 2, Ley 26.934).

Estas leyes confrontan su mirada con la Ley de Estupefacientes 23.737 de 1989 que penaliza todo consumo de sustancias ilegales. Dicha ley sostiene la penalización de la tenencia de drogas. Es decir, que todo aquel que tuviese drogas en su poder y sin importar para qué las tuviera, pasa a ser un criminal plausible de ser detenido y enjuiciado (Basalo, 2017). 
Cabe destacar también que en el marco de los debates de la Ley de Salud Mental se promovieron una serie de cambios que empezaron a resquebrajar o cuestionar las estrategias respecto de las políticas relacionadas con estupefacientes. Uno de los ejemplos más icónicos es la resolución de la Corte Suprema de Justicia de la Nación, del 25 de agosto de 2009. El fallo “Arriola, S. y otros s/causa $\mathrm{N}^{\circ}$ 9080", declaró la inconstitucionalidad de la punición de la tenencia para consumo personal. Dicho fallo se sostiene en los tratados internacionales suscriptos por el país a fin de solicitar a los poderes públicos "asegurar una política de Estado contra el tráfico ilícito de estupefacientes y a adoptar medidas de salud preventivas, con información y educación disuasiva del consumo, enfocada sobre todo en los grupos más vulnerables" (Corda, 2016, p. 16).

Por otra parte, la Ley de Salud Mental avanza en la desmanicomialización de la atención, oponiéndose al encierro, y donde la internación es el último recurso. En contraposición, jerarquiza las modalidades de abordaje interdisciplinarias e intersectoriales, basadas en los principios de la atención primaria de la salud, reforzando la restitución y promoción de los lazos sociales (art.9, Ley 26.657).

Por lo tanto, al priorizar los abordajes bajo los principios de atención primaria de la salud, el contexto y los lazos sociales y comunitarios se pone en evidencia la necesidad de incorporar a los gobiernos locales y a las instituciones territoriales como actores fundamentales a la hora de pensar los abordajes. En consecuencia, nos alejamos tanto de los abordajes punitivos (a cargo de referentes de seguridad) o de los específicos basados en el alejamiento, aislamiento y prohibición del contacto con las sustancias y nos acercamos a otra nueva forma de intervención que plantea la interdisciplina, la intersectorialidad y el trabajo en red, siempre situados en un determinado contexto y territorio. Y es ahí donde las ciudades tienen mucho que decir y aportar.

\section{Coordinación y descentralización, principios para la integralidad de las políticas sociales}

La complejidad inherente a las políticas sociales, en especial las destinadas a problemática de consumo, dan cuenta de la necesidad de que las mismas sean abordadas de forma integral. No existen dudas de que "los problemas sociales se diversifican, se cruzan y no se pueden encerrar en una grilla de categorías cerradas" (Bifarello, 2014). Esto implica trabajar fuertemente en la coordinación interdisciplinaria e intersectorial como medio para lograr la integralidad por un lado y en el fortalecimiento de redes por el otro. Sin embargo, el camino de la coordinación y el de la conformación de redes es largo y muchas veces está minado de dificultades que hacen caer en resoluciones sectoriales o individualistas.

La experiencia de coordinación indica que una de las primeras dificultades que surge es la responsabilidad por el liderazgo, que muchas veces cuesta que sea asumido, así como el quién o quienes convocan al espacio de coordinación. Otra dificultad es cómo se construyen los abordajes entre diversos actores incluyendo la multicausalidad de la problemática, y por último quién (o quiénes) son capaces de "mirar" la trayectoria total o proceso, que estamos abordando, incorporando absolutamente todas las intervenciones y garantizando la comunicación entre ellas. 
En este sentido, resulta esclarecedora la propuesta de Bronso y Repetto (2015) que destacan, a partir del análisis de experiencias latinoamericanas, algunas claves necesarias para la coordinación de políticas públicas. En primer lugar, los autores indican que "la coordinación intersectorial debe servir como instrumento, cuando realmente se necesita, mientras que el logro de los objetivos de política social integral constituye un fin" (Bronso y Repetto, 2015, p. 193) es decir, que no debemos entender la coordinación como un fin en sí mismo. Por otro lado, se entiende que la coordinación resulta costosa, por lo que la misma sólo debe ser requerida "para enfrentar problemas sociales insolubles desde el saber hacer sectorial y que impliquen interdependencia entre los sectores y niveles de gobierno involucrados" (Bronso y Repetto, 2015, p. 193)

Algunos puntos clave para alcanzar la coordinación, a partir de la propuesta de Bronso y Repetto (2015, pp.193-195) son: a) que la rectoría sea asumida por un actor organizacional con alto respaldo político y liderazgo técnico e institucional; b) contar con objetivos claros y metas precisas que brinden un sentido sostenible a los esfuerzos de coordinación, ordenando las prioridades, estableciendo tareas y considerando los plazos de tiempo; c) poseer respaldo de presupuestario, impulsando una formulación de presupuestos que faciliten la integralidad; d) la coordinación debe ser pensada como un sistema, es decir realizar una clara división de tareas entre sectores y niveles de gobierno; e) requiere tanto de la solidez técnica, como de la autoridad política; f) se necesitan instrumentos sólidos de gestión pública, enmarcados en un plan de gobierno general y en la construcción de una intersectorialidad de alta intensidad como un proceso; g) el papel de la ciudadanía es clave, en especial de las comunidades y de las familias en los procesos de formación de las decisiones y en su posterior seguimiento y evaluación; $\mathbf{h}$ ) se requieren de recursos humanos con saberes $\mathrm{y}$ herramientas de gestión transversal, más allá de los saberes sectoriales.

Finalmente, los autores reconocen que para que la integralidad se alcance se requiere que las funciones de coordinación intersectorial influyan sobre las jurisdicciones políticas y administrativas locales. Para ello previamente será necesario contar con los protocolos, instrumentos técnicos y recursos humanos formados en el abordaje de cercanía (Bronso y Repetto, 2015, p. 195)

La experiencia en la ejecución de las políticas sociales, indica que la mayoría de veces un área, un programas o intervenciones aisladas encuentran límites para intervenir, por lo que necesitamos del encuentro con otros y otras más que nunca. Reconocer esto supone un desafío que involucra a los tres niveles de gobierno: nacional, provincial y municipal.

De la experiencia surge el convencimiento de que muchas de estas incertidumbres en relación a los abordajes encuentran su respuesta si se pone el foco en las ciudades y los territorios. No se trata de responsabilizar a los gobiernos locales de toda la complejidad de social que hoy emerge e irrumpe, sino más bien de entender que son los actores adecuados para liderar las coordinaciones, armado de redes locales y poder mirar y continuar las trayectorias que garanticen la integralidad. Lo imprescindible es mostrar que son las ciudades a las que debemos acompañar con los mayores recursos, esfuerzos y un caudal de herramientas pertinentes para fortalecer sus capacidades estatales. 


\section{La experiencia de Santa Fe: la Agencia de Prevención de Consumo de Drogas y Tratamiento Integral de las Adicciones (APRECOD) y el impulso de la red de municipios y comunas}

En marzo de 2016, se pone en marcha en la provincia de Santa Fe la Agencia de Prevención de Consumo de Drogas y Tratamiento Integral de las Adicciones, (APRECOD) en la órbita del Ministerio de Gobierno y Reforma del Estado. La misma fue creada por el gobernador Miguel Lifschitz a través del Decreto 0419/2016 con el principal objetivo de "articular y coordinar acciones con las diferentes jurisdicciones para garantizar el tratamiento integral de los consumos problemáticos de sustancias y las adicciones" (artículo 1, Decreto 0419/2016).

Dentro de los antecedentes de este espacio se destaca la Mesa Intersectorial de Adicciones, conformada en el marco del Gabinete Social de la provincia de Santa Fe, que reunía a los ministerios con el fin de coordinar las políticas sociales para el territorio provincial. Desde este espacio se comenzó a trabajar de forma intersectorial la problemática y en el año 2015 se llevaron adelante capacitaciones para los equipos territoriales de los distintos ministerios sobre el abordaje de los consumos problemáticos de sustancias. ${ }^{2}$

Asimismo, se pueden reconocer diversos esfuerzos que se venían dando desde algunos ministerios para el abordaje de la problemática. En primer lugar, se identifica a la Dirección Provincial de Adicciones, del Ministerio de Desarrollo Social, que contribuía a través de los convenios con organizaciones sociales y derivaciones de tratamiento a las mismas, quedando sus tareas limitadas a tales fines. Segundo, el Programa Lazos, del Ministerio de Educación de la provincia, cuyo objetivo era la prevención y el abordaje integral de las problemáticas de violencias y consumo problemático de sustancias. El mismo había comenzado en 2013 con una prueba piloto en 20 escuelas. Finalmente, en el ámbito del Ministerio de Salud provincial y particularmente desde la Dirección de Salud Mental, se venían sumando esfuerzos para abordar la problemática desde la atención en la salud pública, en simultáneo al proceso de desmanicomialización emprendido en la provincia, en vistas a la aplicación de la Ley Nacional de Salud Mental 26.657.

Reconocidos estos antecedentes, la creación de Aprecod significó un viraje en materia de políticas de drogas en la provincia, dado que asumió el rol como organismo rector y promotor de políticas públicas que aborden la problemática desde el conjunto de los ministerios, resumiendo y coordinando los esfuerzos que ya existían con antelación a su existencia. La inscripción de la Agencia en el Ministerio de Gobierno y Reforma del Estado puso en evidencia el carácter intersectorial que buscó imprimirse en el tratamiento de la política pública, ampliando su alcance y no reduciéndose a un abordaje desde un único aspecto.

En el marco de este trabajo, Aprecod desarrolló el Plan Abre Vida en pos de fortalecer programas provinciales existentes y generar nuevos para dar respuestas efectivas a la situación actual. El plan se divide en tres ejes de acción: prevención, atención integral y tratamiento, e información y comunicación. Para ello se propuso los siguientes objetivos descritos en el Plan: a) prevenir los consumos problemáticos de sustancias psicoactivas; $\mathbf{b}$ ) contribuir al abordaje y tratamiento integral de las situaciones problemáticas de consumo de sustancias psicoactivas; y c) informar y comunicar sobre el estado de los consumos problemáticos y 
las políticas de prevención, cuidado y tratamiento integral desarrolladas por el Estado provincial.

\subsection{Algunos datos sobre el consumo de sustancias psicoactivas en la provincia de Santa Fe}

En Santa Fe, de acuerdo a los datos arrojados por el estudio nacional de población sobre consumo de sustancias psicoactivas (OAD-SEDRONAR, 2017), la sustancia que presenta mayor prevalencia de consumo es el alcohol, seguido por el tabaco y en tercer lugar la marihuana3.

En el diagnóstico que da fundamento al Plan Abre Vida, el Observatorio provincial de Aprecod pone en evidencia que "el consumo de alcohol es el más frecuente: la prevalencia de mes de alcohol es de 55,5\% del total de encuestados. A su vez, si se desagrega este dato por sexo y edad se evidencia que el consumo de alcohol es mayor en varones y en el grupo etario comprendido entre 18 a 24 años" (OAD, 2017).

Es importante destacar que Santa Fe presenta una tendencia similar en sus prevalencias de mes si se las compara con otras provincias de la región centro del país.

\section{Tabla 1.}

Prevalencias (\%) de mes de consumo de sustancias psicoactivas en población de 12 a 65 años en Región Centro y Total País, 2017

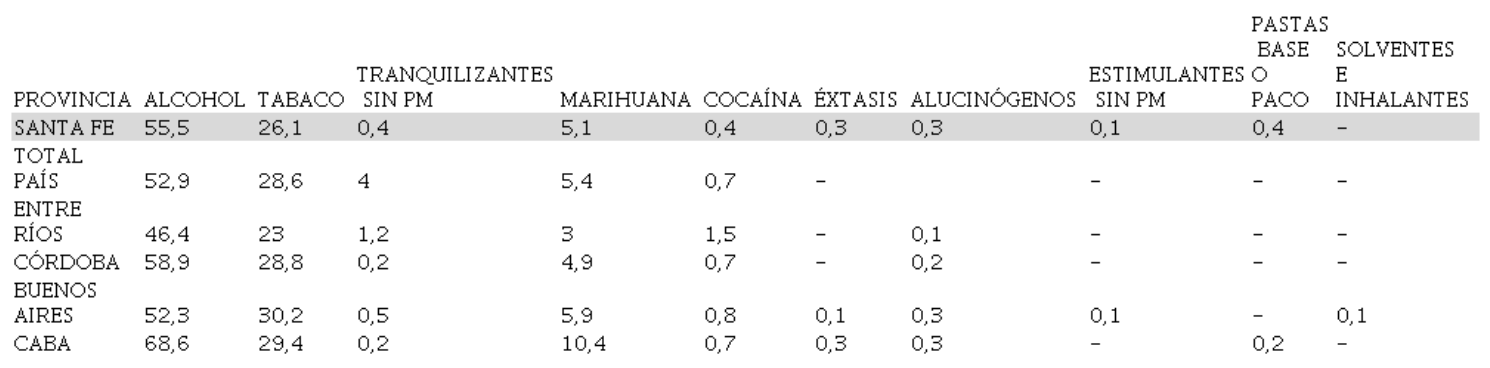

Estudio Nacional de Población de 12 a 65 años sobre consumo de sustancias psicoactivas, Argentina. SEDRONAR, 2017

Como puede advertirse, el consumo de alcohol es una de las problemáticas más relevantes para todas las provincias. Los datos recogidos por el Observatorio provincial del Aprecod a partir del Estudio Nacional de Factores de Riesgo del Ministerio de Salud de la Nación, confirman las prevalencias mencionadas y suman la preocupación por el consumo episódico excesivo4.

Estos datos arrojan uno de los ejes prioritarios de la política provincial, que reproducen una problemática de carácter nacional y mundial tal como se da a conocer en el Informe Mundial de Situación sobre Alcohol y Salud de 2018, que refleja las observaciones realizadas por la Organización Mundial de la Salud. Este organismo muestra la preocupación, no sólo por las numerosas muertes asociadas al consumo excesivo de alcohol, sino también a los factores de riesgo que conlleva como enfermedades no transmisibles, trastornos mentales y comportamentales (Informe Mundial de Situación sobre Alcohol y Salud, 2018 en Plan Abre Vida, 2019). 
Sobre los datos de consumo de alcohol, una de las preocupaciones que se señala para la provincia, en similitud al contexto nacional, es el impacto que tiene sobre los jóvenes, principalmente entre aquellos de 18 y 24 años, seguido por el grupo de 25 a 34 años.

Asimismo, si se mira otro de los indicadores arrojado por la encuesta de Sedronar, se da cuenta de que la edad de inicio del consumo de alcohol es de 16 años en varones y de 17 años en mujeres; mientras que la edad de inicio para el tabaco se visualiza a los 15 años para ambos sexos. Estas sustancias son las de consumo a más temprana edad, seguidas por la marihuana (19 años) y la cocaína (20 años). Al comparar la provincia de Santa Fe con el total del país se observa una tendencia similar en relación a la edad de inicio del consumo de sustancias psicoactivas.

Los datos arrojados por los estudios mencionados, invitan a poner el foco en el consumo de alcohol. Asimismo, las prevalencias de consumo de sustancias, principalmente de alcohol en edades tempranas, que también se verifican en el indicador de la edad de inicio, dan justificación a la prioridad puesta en niños, niñas y adolescentes, así como programas que focalizan la prevención en jóvenes.

$\mathrm{Al}$ mismo tiempo, estas políticas son prioritarias para el cumplimiento de la meta 3.5 de los Objetivos del Desarrollo Sostenible del Programa de las Naciones Unidas para el Desarrollo que buscan "Fortalecer la prevención y el tratamiento del abuso de sustancias adictivas, incluido el uso indebido de estupefacientes y el consumo nocivo de alcohol".

\subsection{Territorio y descentralización: la conformación de la Red de Municipios y Comunas}

La extensión del territorio santafesino presenta un desafío en materia de implementación de una política pública, dado que posee $720 \mathrm{~km}$. de norte a sur y $380 \mathrm{~km}$ en su eje este a oeste, con diversidades no sólo geográficas, sino también en su estructura social y productiva.

Otra de sus características destacadas es que, junto con Córdoba, es una de las provincias más municipalizada del país: está dividida políticamente en 19 departamentos que agrupan 365 localidades: 55 municipios, es decir, localidades de más de 10.000 habitantes y 310 comunas, localidades de menos de 10.000 habitantes, cada una de las cuales posee un gobierno electo por sus ciudadanos.

Asimismo, se caracteriza por la distribución desigual de su población, que suman un nuevo reto en la implementación de una política pública. Por una parte se pueden identificar dos grandes conglomerados urbanos. La ciudad de Rosario, que según el censo 2010, cuenta con 1.198.528 habitantes, representando un $37,4 \%$ de concentración de la población provincial, y al mismo tiempo, es la tercera ciudad más poblada de Argentina. Con una distancia significativa, le sigue Santa Fe de la Vera Cruz (484.000 habitantes) capital de la provincia, la cual reúne un $16,14 \%$ de la población santafesina.

Una característica diferente presentan un conjunto de ciudades intermedias: Rafaela (93.000 hab.), en el noroeste; Venado Tuerto (82.000 hab.) al sur y Reconquista (77.000 hab.) ubicada en el norte provincial. Las mismas se han establecido como centros del desarrollo territorial de la provincia, siendo seleccionadas como ciudades "nodo" en el proceso de descentralización que se ha 
ido desarrollando desde 2008. Otras ciudades que presentan gran concentración urbana se extienden en las regiones metropolitanas de las dos grandes urbes, como por ejemplo, Villa Gobernador Gálvez (78.000 hab.), parte de la región metropolitana de Rosario y Santo Tomé (66.000 hab.), lindera a la capital santafesina.

La implementación del Plan Abre Vida en el territorio santafesino puso en evidencia la necesidad de generar alianzas estratégicas con diversos actores, a fines de garantizar su alcance territorial. El informe 2019 de Aprecod reconoce "La implementación del Plan Abre Vida es posible gracias a la articulación de una pluralidad de actores que se coordinan desde Aprecod a nivel interdisciplinario, intersectorial e interjurisdiccional"

El Plan Abre Vida fue presentado en la provincia en enero de 2017, en abril del mismo año, se hizo una primera convocatoria a los gobiernos de Municipios y Comunas a adherirse a una red de trabajo que fue presentada bajo el nombre de "Red de Municipios y Comunas para la prevención y abordaje integral de los consumos problemáticos de sustancias"

El documento de adhesión presenta como principal objetivo de la red el de impulsar políticas públicas que permitan la promoción de la salud, la prevención de los consumos problemáticos de sustancias en la población en general a fines de promover no sólo el tratamiento sino también la integración de las personas que atraviesan situaciones de consumo. Asimismo, el documento prioriza el acompañamiento de niños, niñas y adolescentes.

Desde 2017, la red provincial fue sumando la adhesión de los gobiernos locales. Para fines de 2019 cuenta con 112 gobiernos locales adheridos: 33 municipios y 79 comunas. 


\section{LOCALIDADES ADHERIDAS A LA RED DE MUNICIPIOS Y COMUNAS DE APRECOD}

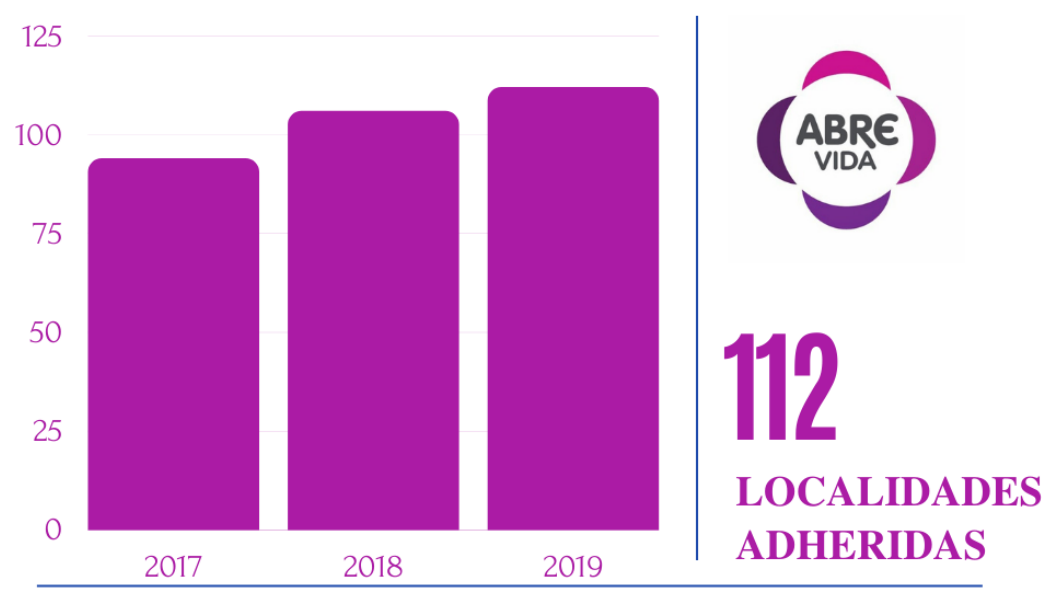

Imagen 1.

Número de localidades adheridas a la Red de Municipios y Comunas de Aprecod Elaboración propia a partir de informe de gestión de Aprecod 2017-2019

Esta primera acción desarrollada por la red debe ser leída como clave para el proceso de agendación de la temática a los gobiernos locales. Significó por un lado la visibilización de la problemática y su agendación en la política local. Asimismo, simbolizó un compromiso político asumido por los responsables de la política provincial y los gobiernos locales. Finalmente, el acto de firma fue el impulso a la construcción de una red de trabajos y la apertura de canales de comunicación entre la provincia y los municipios.

\subsubsection{Herramientas para la descentralización}

La primera instancia para el fortalecimiento de los gobiernos locales en el abordaje de la problemática, estuvo dada por visitas técnicas y encuentros de capacitación que buscaron imprimir la mirada desde el paradigma de la complejidad y recuperar las experiencias que los municipios y comunas habían desarrollado para el abordaje de la problemática, reconociendo situaciones que iban desde el abordaje a través de las áreas sociales o equipos de salud; a experiencias en las que se habían conformado equipos específicos para el abordaje de la problemática (Pérez) o incluso proyectos de centros de día municipales (Rosario; Avellaneda). 
Asimismo, en el marco del Plan Abre Vida se reconocen dos programas que resultaron clave para la extensión territorial del plan y vertebradores de la política preventiva por un lado y del eje de atención integral por el otro.

En primer lugar, en el ámbito de la prevención, la coordinación con el Programa Lazos del Ministerio de Educación de la Provincia de Santa Fe, que pasó a tomar el nombre de Lazos-Abre Vida tomó un impulso en el cual se extendió a "700 escuelas nivel Nivel Inicial, Primario y Especial (IPE) con proyectos institucionales de prevención de consumos problemáticos; 350 escuelas secundarias con Consejo de Convivencia Escolar; 50 instituciones para Adultos y Nivel Superior" (Aprecod, 2019). Asimismo, a partir de la construcción del dispositivo "Construyendo Lazos", una obra de teatro que sensibiliza a sobre los consumos y los vínculos entre pares y con adultos referentes permitió el nexo entre las escuelas y las comunidades, dado que se dirige no sólo a los jóvenes, sino a sus familias e instituciones de la localidad. "De 2017 a 2019 se realizaron 37 jornadas Construyendo Lazos en 20 localidades, con la participación de 7265 jóvenes y adultos” (Aprecod, 2019). Por otro lado, la realización de una jornada de formación bajo el programa Escuela Abierta del Ministerio de Educación, en el año 2017 permitió alcanzar a la totalidad de los docentes y jóvenes de escuela secundaria brindando una primera aproximación a la problemática.

En segundo lugar, en materia de atención integral de la problemática, la puesta en marcha de los Centros de Referencia y Orientación "Equipo de Enlaces" de Aprecod, resultaron ordenadores para la conformación de redes para el abordaje, facilitando el acercamiento entre actores: ciudadanía, equipos sociales locales, equipos de salud de los distintos niveles y las 29 organizaciones de la sociedad civil conveniadas bajo el programa "Redes de Cuidado" que brindan "prestaciones para el cuidado y tratamiento de personas sin obra social/prepaga que atraviesan situaciones de consumos problemáticos de sustancias" (Aprecod, 2019).

La puesta en marcha de estos programas permitieron visibilizar la importancia de los territorios como espacios de coordinación de las políticas públicas, y el rol de Aprecod como facilitador y promotor de la red.

\subsubsection{Fortalecimiento de proyectos preventivos, a través de la descentralización de recursos}

A partir de la segunda mitad del año 2017 se puso en marcha otro componente del programa de la red que permitió la transferencia de recursos a los municipios y comunas para el desarrollo de proyectos de prevención específicos.

Para la presentación de los proyectos se propuso un formulario abierto que permita a las localidades presentar propuestas que den cuenta del diagnóstico en ese territorio en específico y articular respuestas acordes, entendiendo que la problemática se presenta de forma diversa en cada localidad Se entiende que: "Cada territorio debería diseñar sus políticas teniendo en cuenta sus particularidades históricas, idiosincráticas y culturales, así como su propio contexto y problemáticas. Diferentes drogas que genera diferentes daños en diferentes contextos (y agregaría a diferentes actores), seguramente requerirán diferentes intervenciones" (Gonzalez et.al, 2017, p. 5) 
Para los proyectos, se solicitó a los municipios, como requisito el cumplimiento de los marcos legales nacionales y provinciales y la coherencia entre diagnóstico y propuesta, en conjunto con un cronograma de actividades realizable en el tiempo de duración del proyecto. Asimismo, se les pidió reconocer actores de su territorio que sean clave para el desarrollo de la política territorial e integrarlos en sus propuestas.

La presentación de los proyectos sorprendió con una amplia variedad de estrategias, algunas de las cuales se centraron en los espacios de prevención inespecífica, otros de prevención específica y otros en la puesta en marcha o consolidación de centros de atención y orientación territorial, que pusieron el foco en el eje de la atención.

En total en el año 2019 hay 18 proyectos financiados a lo largo del territorio provincial, que alcanzan un presupuesto de $\$ 5.478 .000,00$ (Aprecod, 2019).

Sólo a modo de ejemplo podemos mencionar tres experiencias diferentes sobre las estrategias utilizadas por los gobiernos locales.

En la ciudad de Avellaneda, en el norte de la provincia de Santa Fe, el financiamiento se utilizó en pos de fortalecer el Centro de día Municipal. En el mismo se brinda contención a jóvenes de la ciudad a través de propuestas lúdicas, formativas y promoviendo espacios de promoción de la salud. El proyecto del centro de día era anterior a Aprecod, y se vio la necesidad de fortalecerlo con la incorporación de nuevos recursos humanos.

En la ciudad de Villa Gobernador Gálvez, a diferencia de la experiencia anterior, con el financiamiento otorgado se puso en funcionamiento un equipo, al que se denominó "equipo matricial", que tiene como fin generar redes en la ciudad para la atención de la problemática, sirviendo como nexo y soporte de las instituciones existentes: centros de día, centros de salud, hospital, escuelas, etc.

Una tercera experiencia la constituye la del municipio de Pérez, el cual desde 2013 cuenta con el Centro de Asesoramiento y prevención en Consumos Problemáticos. En este caso el recurso humano que se suma a partir del financiamiento de la Agencia permitió pensar nuevas estrategias armando un espacio de bajo umbral que permitió la contención de aquellos usuarios que, por diversas situaciones, no podían sostener otros tratamientos para la atención de su salud.

Las restantes experiencias se centran sobre todo en la puesta en marcha de espacios de prevención inespecífica y específica, sobre todo destinados a jóvenes que tienen como objetivo principalmente la suma de factores protectores y la reconstrucción de lazos sociales.

De 2017 a 2019 se financiaron 47 proyectos, de 18 localidades, alcanzando una inversión de 9.891.000,00 de pesos (Aprecod, 2019). ${ }^{5}$

\subsection{Municipios en Acción: coordinación Nación, Provincia y municipios para el desarrollo de planes locales}

En 2018 la provincia adhirió al programa nacional Municipios en Acción, que lleva adelante la Sedronar. El mismo se propone acompañar a los municipios en la "implementación, seguimiento y evaluación de planes locales sobre consumos problemáticos que permitan trabajar sobre la problemática de las adicciones atendiendo las particularidades de cada intendencia" (sitio web de Sedronar, s/f). 
La existencia previa de la red provincial permitió un alcance amplio del programa en corto plazo, resultando en que 80 localidades respondan y se sumen al programa nacional. Esto posicionó a Santa Fe como la provincia con mayor cantidad de localidades adheridas y donde el programa se ha instalado de forma más rápida. En este sentido, se destacó la presencia de los tres niveles de gobierno en el despliegue del programa.

La primera instancia implicó la capacitación de equipos técnicos municipales en la elaboración de diagnósticos y en el diseño de un plan local de las cuales participaron 201 miembros de equipos locales. Finalizado el proceso de capacitación, 32 municipios desarrollaron sus planes locales.

Este núcleo de capacitaciones se suma a la oferta brindada a través de la Agencia y Sedronar sobre paradigmas de abordaje, prevención comunitaria, atención primaria de la salud, etc. que contribuyeron a instalar la temática.

El desarrollo del programa Municipios en Acción en el territorio santafesino, y su fuerte acento en el desarrollo de planes sirvió a fines de lograr una meta compartida, pasar de acciones de tipo reactivas, a poder pensar una planificación de acciones vinculadas a la temática; en este sentido, esto contribuiría a pasar de gestiones con el acento puesto en la atención de casos individuales (demandas), a poner el foco en la prevención de los consumos problemáticos, llegando a un mayor número de ciudadanos.

\section{Reflexiones finales}

No hay dudas que las problemáticas asociadas a los consumos de sustancias psicoactivas irrumpen en la agenda de las políticas sociales y urbanas como problemas complejos que demandan respuestas integrales. Si bien existe una coincidencia en los organismos internacionales y los estudios de la temática sobre la necesidad de un abordaje intersectorial, multinivel y desde los derechos humanos, aún son pocas las experiencias analizadas que, desde el estudio de las políticas públicas, discutan sobre la implementación de estos nuevos enfoques, y se reducen aún más si nos acercamos al plano subnacional.

La experiencia santafesina de la Aprecod y la conformación de la Red de Municipios y Comunas para la prevención y abordaje de los consumos problemáticos puede contribuir al debate, aun reconociendo que se trata de una experiencia acotada en el tiempo.

Se reconocen algunas fortalezas del ejercicio y las herramientas arriba descritas. En primer lugar, la Red propició la vinculación entre los tres niveles del Estado y con la sociedad civil, tomando al territorio como escenario de ese encuentro. Segundo, se destaca el lugar de la Agencia y el gobierno provincial como articulador y coordinador de la red. Si bien sería deseable que fueran los mismos municipios los impulsores de la coordinación, en la actualidad, la instancia provincial ha sido la principal actora en términos de gobierno de la red. Tercero, las capacitaciones y asesoramientos técnicos han contribuido a la instalación de herramientas, no sólo sobre la temática -impulsando el paradigma desde la complejidad-, sino también vinculados a la gestión, como por ejemplo el diseño de planes locales. Cuarto, el programa ha tenido la suficiente flexibilidad para adaptarse a contextos diferentes, no presentándose como un dispositivo estanco y cerrado, sino como un marco de trabajo. Asimismo, se destaca que desde los 
tres ámbitos estatales se asumieron compromisos políticos, que acompañaron el desarrollo técnico de las iniciativas. Resulta interesante señalar que estos puntos coinciden con algunos de los señalados por Bronso y Repetto (2015) para las experiencias de coordinación, entendiéndose como un camino que contribuye a la integralidad.

Entre las principales desafíos a profundizar se reconoce que no se ha podido alcanzar a la totalidad del territorio provincial y que la descentralización en términos de recursos financieros de transferencia directa alcanzó a un número pequeño de localidades; se replica en este sentido, una de las principales dificultades señalada por Subirats y Montaner (2012), que es la excesiva dependencia de los gobiernos locales, principalmente por la escasez de recursos.

Finalizando, consideramos que la principal riqueza de la experiencia señalada es la valorización de la ciudad como escenario de políticas urbanas, que son el resultado de la interacción de decisiones y de acciones de muchos niveles y de muchas esferas de gobierno. Consideramos que esta primera aproximación a la experiencia puede servir como una puerta para seguir reflexionando y contribuir al desarrollo de herramientas teóricas, metodológicas e instrumentales necesarias para abordar el tratamiento de la problemática de los consumos de sustancias psicoactivas.

\section{Referencias}

APRECOD. (2017). Documento Orientativo para el Abordaje de los Consumos problemáticos. Santa Fe, Gobierno de Santa Fe.

APRECOD (2019). Informe de gestión 2017-2019, sin publicar.

APRECOD (2019). Plan Abre Vida 2017-2019, sin publicar.

APRECOD (2019). Sistematización de principales indicadores sobre consumos problemáticos en la provincia de Santa $F e$, en base a Estudio Nacional en Población de 12 a 65 años sobre consumo de sustancias psicoactivas, Argentina, SEDRONAR, 2017, sin publicar.

Asociación Civil Intercambios, Biblioteca http://intercambios.org.ar/es/biblioteca.

BASALO, G. (2017). Despenalizar la tenencia de drogas es respetar los derechos humanos. En Touzé. G., (comp.), Avances y retrocesos en políticas de drogas, Conferencias Nacionales sobre Politicas de Drogas 2010-2017. CABA: Intercambios Asociación y Universidad de Buenos Aires, Facultad de Ciencias Sociales.

BIFARELLO, M. (2014). Políticas sociales: problemas complejos, respuestas integrales. En: BERETTA, D.; GALANO, N., Solos no se puede: Política social, actores y estrategias de gestión. Rosario: Laborde Libros Editor.

BRONSO, C.; REPETTO, F. (eds.) (2015). Coordinación de politicas sociales: desafíos para la gestión pública. Madrid: Estudio nro.18, Programa EUROsociAL.

CORDA, R. (2016). La estrategia fallida: encarcelamientos por delitos relacionados con estupefacientes en la Argentina. Buenos Aires: Intercambios Asociación Civil, Universidad de Buenos Aires. Facultad de Ciencias Sociales.

Decreto No 0419 (2016). Creación de Agencia de Prevención Del Consumo de Drogas y Tratamiento Integral de las Adicciones (Aprecod) 
DEFENSORÍA DE NIÑOS, NIÑAS Y ADOLESCENTES (2016). Aportes sobre el consumo problemático de sustancias psicoactivas para una politica pública integral destinada a niños, niñas y adolescentes. Santa Fe: Gobierno de Santa Fe.

Gobierno de la Provincia de Santa Fe. Sitio web oficial. www.santafe.gov.ar

GONZALEZ, P. et al. (2017). Un libro sobre drogas. CABA: El Gato y la Caja.

REPETTO, F. (2009). Coordinación de Políticas Sociales: Abordaje Conceptual y Revisión de Experiencias Latinoamericanas. Presentación en el XIV Congreso Internacional del CLAD sobre la Reforma del Estado y de la Administración Pública, Salvador de Bahía, Brasil, 27 al 30 de octubre.

Ley Nacional N 23.737 (1989). Tenencia y Tráfico de Estupefacientes.

Ley Nacional N 26.657 (2010). Ley Nacional de Salud Mental.

Ley Nacional $N^{\circ} 26.934$ (2014). Plan Integral para el Abordaje de los Consumos Problemáticos.

Ley Nacional No 23.849 (1990). Aprobación de la Convención de los Derechos del Niño.

Ley Nacional N² 26.061 (2005). Protección Integral de los Derechos de las Niñas, Niños y Adolescentes

Ley Provincial No 10.772 (1991). Ley Provincial de Salud Mental

Ley Provincial No 12.967 (2009). Promoción y Protección Integral de los Derechos de las Niñas, Niños y Adolescentes

ONU (1948). Declaración Universal de Derechos Humanos.

Plan Abre Vida. Sitio web oficial. Gobierno de Santa Fe, www.santafe.gov.ar/abrevida

REPETTO, F.; FERNÁNDEZ, J.P. (2012). Coordinación de politicas, programas y proyectos sociales. Buenos Aires: Fundación CIPPEC.

Secretaría de Políticas Integrales sobre Drogas. Sitio Web Oficial. https://www.argent ina.gob.ar/sedronar

SEDRONAR (s/f). Orientaciones para el Abordaje de los lineamientos curriculares para la prevención de las adicciones. CABA: Presidencia de la Nación.

SEDRONAR. (2018). Municipios en Acción. Fundamentos y Estrategias para una politica local sobre drogas. CABA: Presidencia de la Nación.

SUBIRATS, J.; MONTANER, J. (2012). Perspectivas de cambio y nueva mirada sobre la realidad urbana. En: SUBIRATS, J.; MONTANER, J. (comp.), Repensar las politicas urbanas. Apuntes para la agenda urbana. Barcelona: Ediciones Diputació de Barcelona.

\section{Notas}

1 La Sedronar en su Manual de Orientaciones para el abordaje, brinda un ejemplo que pone luz a la necesidad de comprender los tres factores: "si una persona adulta toma dos copas de vino (sustancia) en una fiesta, ¿Se puede pensar que esto es problemático? Si a esto se le suma que llegó cansado y sin haber comido y al finalizar la fiesta, vuelve a su casa manejando su auto (contexto), ¿Puede pensarse que su consumo se vuelve problemático? Si nos centramos solamente en la sustancia, podríamos decir que no. Pero si tenemos en cuenta el sujeto, la sustancia y el contexto, y la relación entre estos factores, la respuesta podría ser otra" (Sedronar s/f:13)

2 El Gabinete Social del gobierno de la Provincia de Santa Fe se puso en funcionamiento en 2008, a partir del Decreto 0063. Tiene por objetivo "elaborar la estrategia integral de gobierno destinada a apoyar y beneficiar a las personas y grupos familiares más desprotegidos, con la finalidad de erradicar la extrema pobreza del territorio 
provincial". En una primera etapa fue coordinado por el Ministerio de Gobierno y Reforma del Estado y estuvo conformado por el Ministerio de Desarrollo Social, Ministerio de Salud, Ministerio de Educación, Ministerio de Trabajo y Seguridad Social, Ministerio de Innovación y Cultura en carácter de miembros permanentes. En 2012, el Ministerio de Desarrollo Social comienza a coordinar el espacio y se suman el Ministerio de Seguridad, Ministerio de Justicia y Derechos Humanos, Ministerio de Aguas, Servicios Públicos y Medio Ambiente; y la Secretaría de Estado de Hábitat. (Sitio web de la provincia de Santa Fe)

3 Las medidas de prevalencia indican el uso de las sustancias efectuado por una persona entendida como consumidor. Se diferencian tres tipos de prevalencias: a) prevalencia de los últimos 30 días o del mes, es la proporción de personas que consumieron la sustancia en los últimos 30 días sobre el total de las personas estudiadas. b) Prevalencia de los últimos 12 meses o anual, proporción de personas que consumieron la sustancia en los últimos 12 meses sobre el total de las personas estudiadas. c) Prevalencia de vida o global:, proporción de personas que consumieron la sustancia alguna vez en la vida sobre el total de las personas estudiadas.

4 El consumo episódico excesivo es definido como el consumo de 5 tragos o más en una misma oportunidad en los últimos 30 días

5 Las ciudades que recibieron financiamiento a través de este programa son: Avellaneda, Reconquista, Villa Ana, Sunchales, Frontera, Rincón, Recreo, Monte Vera, San Justo, Laguna Paiva, Arroyo Leyes, Gálvez, Cayastá, Franck, Villa Gobernador Gálvez, Pérez, Carcaraña, El Trébol y Firmat.

\section{Notas de autor}

Sobre la autora

Cecilia Nieto es Lic. en Comunicación Social (UNR). Docente y Sub coordinadora del grupo de estudio en Políticas Sociales Urbanas de la Fac. de Ciencia Política y Relaciones Internacionales (UNR). En los últimos 20 años ocupó distintos cargos en áreas de cooperación internacional, planificación y políticas sociales tanto en la gestión local como provincial. Cursa actualmente la maestría de Desarrollo Humano de FLACSO.

Sobre la autora

María Laura Sartor Schiavoni es Lic. en Ciencia Política (UNR) Candidata a magister de la Maestría de Estudios Electorales (UNSAM). Es docente y miembro del grupo de Políticas Sociales Urbanas de la Fac. de Ciencia Política y Relaciones Internacionales (UNR). Trabajó en distintas áreas de políticas sociales del Gobierno de Santa Fe y actualmente se desempeña en el área de participación ciudadana de la Municipalidad de Rosario. 